\title{
SMART HOSPITAL TECHNOLOGY
}

\author{
${ }^{1}$ M J Jayashree , ${ }^{2}$ Aju Sam Sunny, ${ }^{3}$ Anu John, ${ }^{4}$ Ashley Anna Sunny, ${ }^{5}$ Sruthi Susan \\ Sam. \\ Department of Electronics \& Communication Engineering,Mar Baselios College of \\ Engineering and Technology,Mar IvaniosVidya Nagar, Bethany Hills , Nalanchira \\ Thiruvananthapuram -15, Kerala, India
}

\begin{abstract}
The ECG signals captured from the body of the patient using three electrode model is processed and conditioned by the analog front end device is finally sent to the data acquisition unit. The data acquisition unit used is the user pc/ laptop with MATLAB. Using very specific image processing techniques the critical intelligence from the captured image is extracted. From this processed image any sort of abnormal conditions is determined which is informed to the corresponding doctor via text message. Simultaneously the processed image is sent to the doctor mail by using specific TCP/IP protocol.
\end{abstract}

\section{KEYWORDS}

DFT, FFT, IFFT, LPF, Arduino

\section{INTRODUCTION}

In Telemedicine communication through audio and video is done to convey or exchange the details of a patient with a physician. Using this process the details of a patient can also be discussed and analysed. In health information technology Telemedicine plays a major role. Telemedicine is basically the combination of medicine \& information technology. This involves the transmission of medical information electronically. Hence this can be used for conveying the condition and status of patient to a doctor if the doctor is not available near the patient. Different methods can be applied for this purpose For example: store-and-forward technology, secure messaging; e-mailing,

By monitoring the data received, the doctor is able to understand the patient's condition, to diagnose the disease and to take remedial measures. Videoconference is an effective measure in this case.

\section{TELE MONITORING}

Tele monitoring is the monitoring patients who are not at the same location as the doctor. For example consider a patient with monitoring devices at home. He can easily transmit the results of to the doctor through telephone. This assists the patient to perform the initial and basic stage of health care by himself. Also this avoids the un necessary travel by the patient. Tele medicine is a fast growing field. A new type of Tele monitoring known as primary remote diagnostic disease has been introduced in a number of developing countries. In this technique devices are used by the doctor for the examination, diagnosis and treatment remotely. This type of treatment

DOI: $10.5121 /$ hiij.2014.3302 
is mainly used for monitoring the chronic diseases which have been diagnosed already. But this new technology can also be applied for monitoring and diagnosing the common diseases such as blood pressure, blood glucose, heart rate, weight etc. Frequent monitoring of the patient's condition enables the physician to suggest the best and effective course of treatment to the patient.

\section{COMPONENTS OF THE SYSTEM}

The system comprises of signal acquisition unit, processing unit and alerting system. The signal acquisition unit is the high precision analog front end IC named AD620A from Analog devices. It is a low cost, high accuracy instrumentation amplifier that requires only one external resistor to set gains from 1 to 1000. The low noise, low input bias current and low power of the AD620 make it well suited for medical applications such as ECG and non-invasive blood pressure monitors.

For the simulation of the below mentioned image processing algorithm, we use ECG signal which is captured from the body in real time. The processing unit is coupled with a PIC micro controller which acts as the alerting system. Data processing unit comprises of user PC / Laptop loaded with MATLAB.

\section{Image Processing}

The atrial or ventricular regularity or irregularity is a measure of the cardiac rhythm which in turn gives an idea about the regularity or irregularity of the heart beat. The consistency of patterns between the $\mathrm{p}$ waves accesses atrial regularity while the consistency of patterns between $\mathrm{R}$ waves accesses ventricular regularity. Typical ECG waveform is shown in Fig.No.1
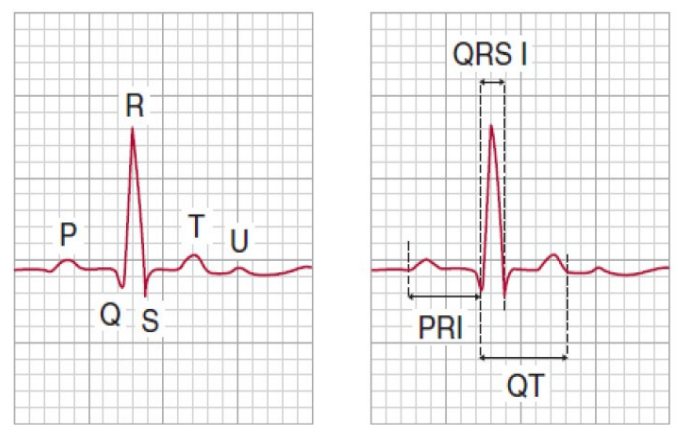

Fig.No.1: Typical ECG waveform

Initially eyeballing, the rhythm for regularity was used. Now two more methods are available, one is the use of callipers and the other is paper technique. In Callipers two needle points are hinged together, one placed at the peak of the $\mathrm{P}$ wave or $\mathrm{R}$ wave and the other placed at the peak of the subsequent $\mathrm{P}$ wave or $\mathrm{R}$ wave. The needle points are held steadily and the callipers are moved down the strip. The needle points of the calliper if fall at the peaks of the subsequent $P$ waves shows atrial regularity. If the needle point falls on the peaks other subsequent $\mathrm{R}$ waves shows ventricular regularity. On the other hand if the needle point do not fall at the peaks of the $\mathrm{P}$ waves or $\mathrm{R}$ waves shows atrial and ventricular irregularities respectively. 
In paper technique a clean and straight edge of paper is lined up with the peak of the $\mathrm{P}$ waves or $R$ waves. Three $P$ wave peaks in a row are marked on the edge of the paper and the paper is then moved to three subsequent $\mathrm{P}$ wave peaks. If the initial marks match with the marks of the subsequent $\mathrm{P}$ waves that shows atrial regularity and if there is no matching between the marks it shows atrial irregularity. Similarly ventricular regularity or irregularity is determined by repeating the process with Rwaves.

\section{IMPLEMENTATION ALgORITHM}

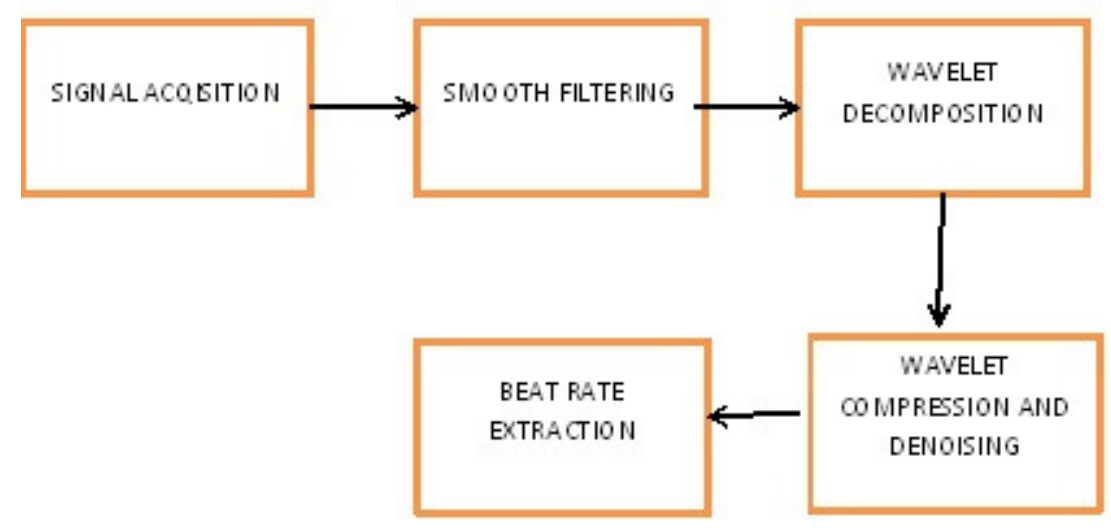

Fig.No.2 Block diagram of Algorithm

\section{Step1:}

Obtain ECG signal from the controller, which is in digital form. The digital values obtained in the controller is collected in MATLAB software in real time.

\section{Step 2:}

Apply smooth filtering process to remove higher frequencies. The most randomly occurring signals are eliminated by setting a threshold value. These high frequency signals do not contribute any intelligence. It may be due to loose contacts in the electrode or variations in impedance matching.

\section{Step 3:}

To extract vital information from the ecg image apply wavelet decomposition technique.It is done by using the function 'wavedec'.

\section{Step 4:}

Set a threshold value for identifying the critical condition in heart beat rate using the relation (maximum value-mean value) / 2 .

\section{Step 5:}

R peak detection: Done using the function 'waverec', inverse of 'wavedec', which performs a multilevel one dimensional wavelet reconstruction.

\section{Step6:}

If the adjacent peak distance is greater than a specific value, save the corresponding image to a particular location in the computer.

Step7:

Calculate heart beat rate. 
From the final detected peaks any sort of abnormality can be easily detected. In case of emergency the PIC controller is triggered and the status of the patient is sent to the doctor as text message using a GSM modem as shown in the block diagram given below.

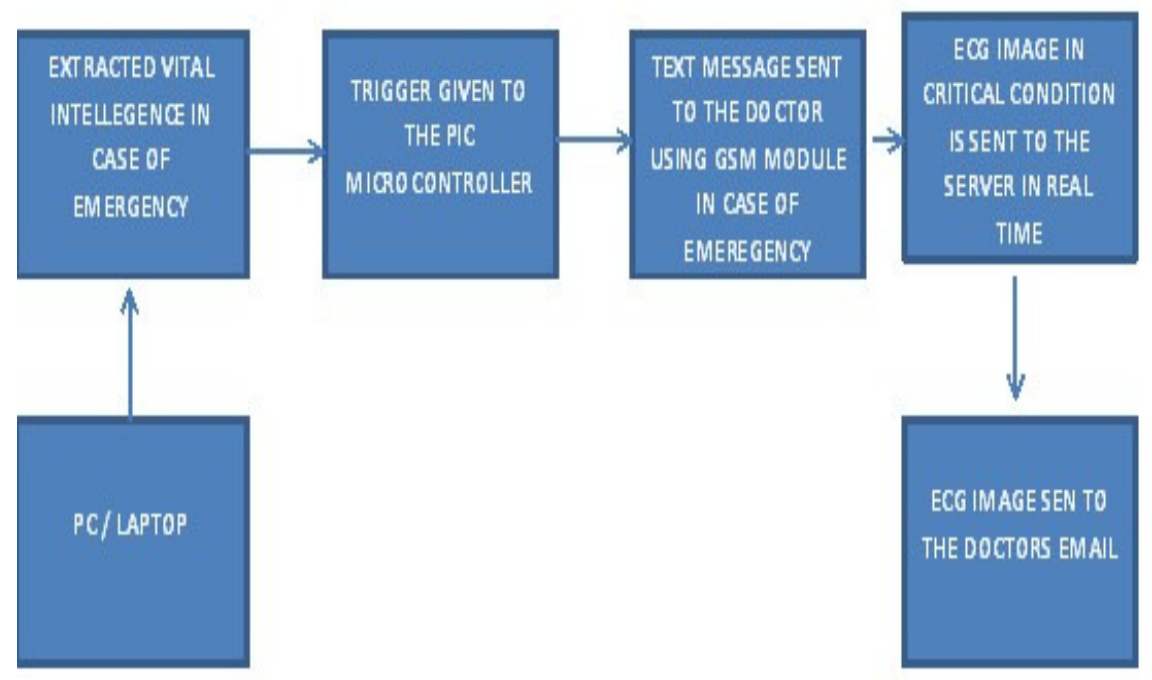

Fig No 3. Block diagram representation of emergency alerting system

\section{Simulation RESUltS}

The setup is as shown in the Fig No.4

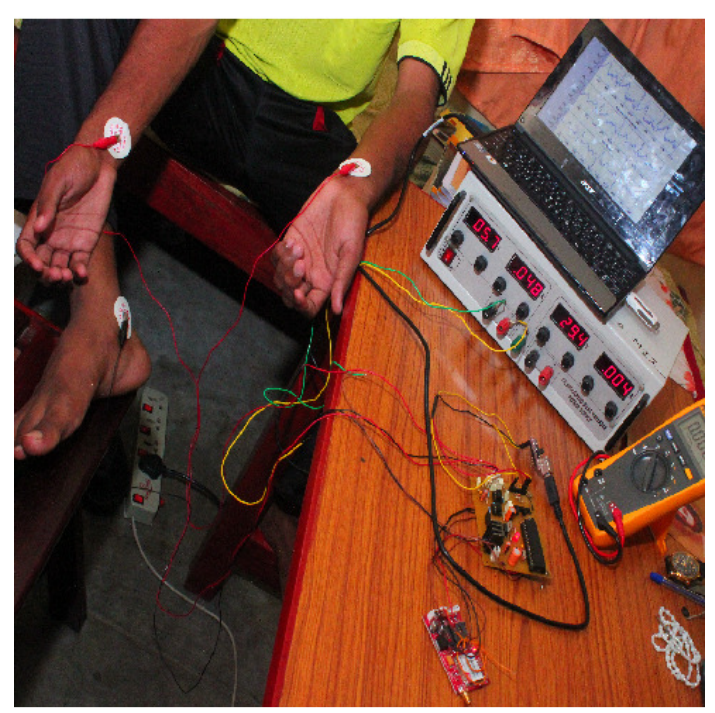

Fig No 4. The system 

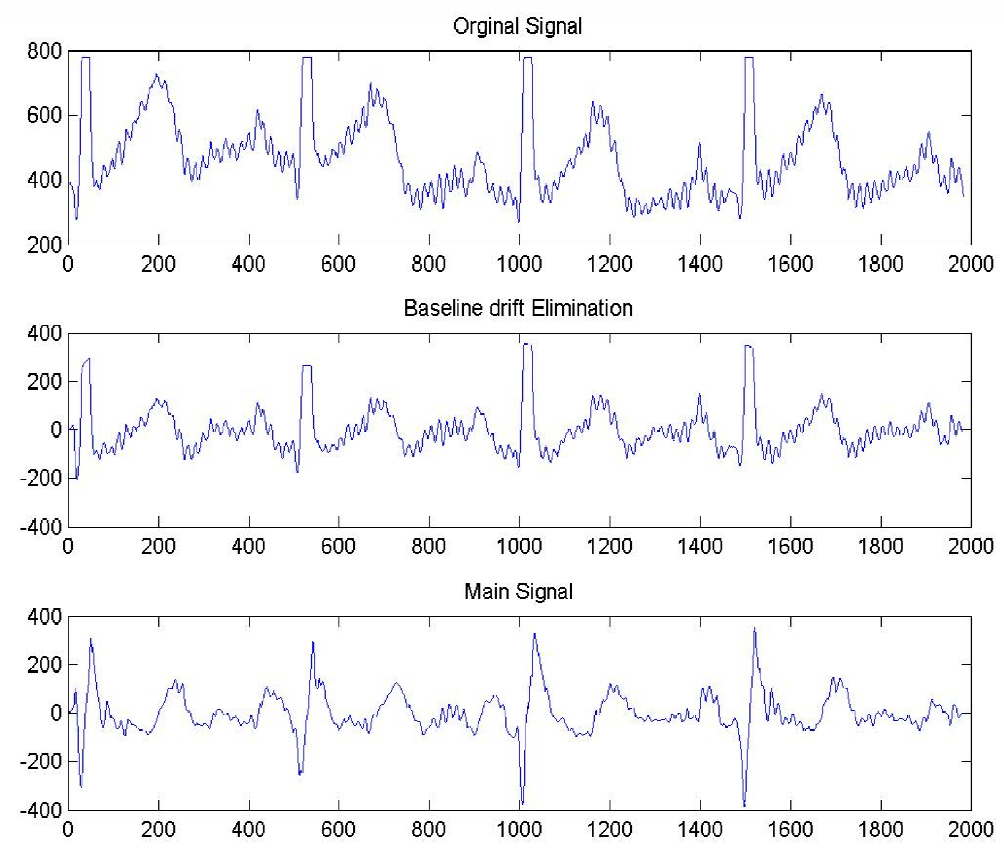

Fig No 5 Final detected peaks

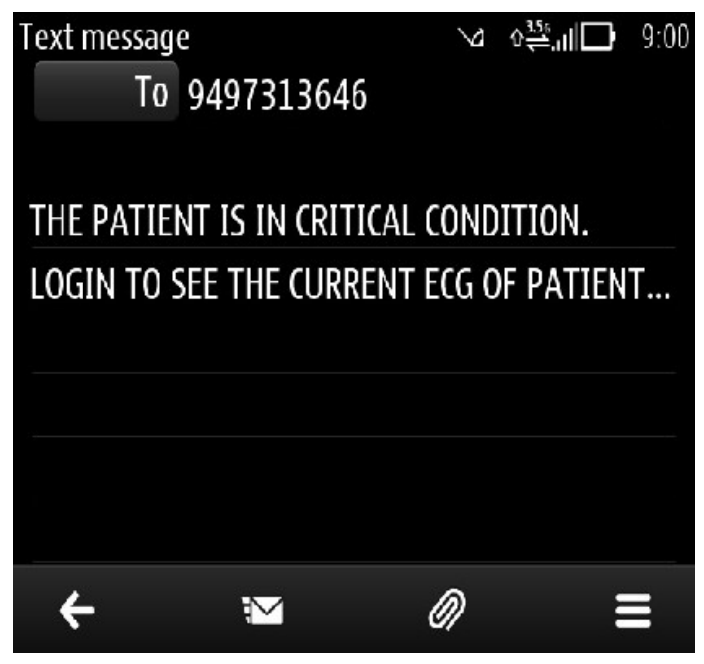

Fig No.6.Screen shot of message on doctor's handset

\section{EMERgEnCY Alerting SYSTEM}

The extracted vital intelligence in case of emergency is uploaded to the server. The image which is now saved in the server is automatically sent to the mail address of the doctors. In case of 
emergency the trigger is given to PIC controller which will send text message to the doctor using GSM modem. From the server the critical ECG image is sent to doctor's email address.

For the website creation Microsoft Visual Studio 2010 is the IDE been used .The programming language used here is $\mathrm{C \#}$.

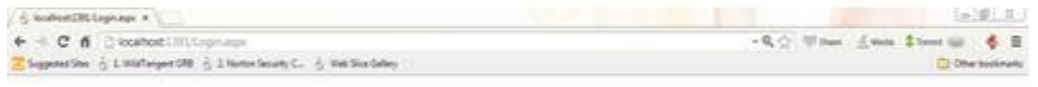

\section{SMART HOSPITAL}

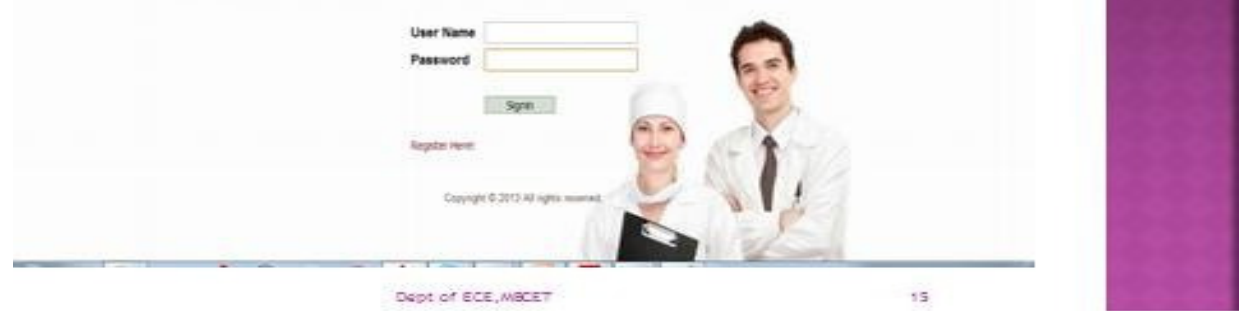

Fig No.7.Login page of website

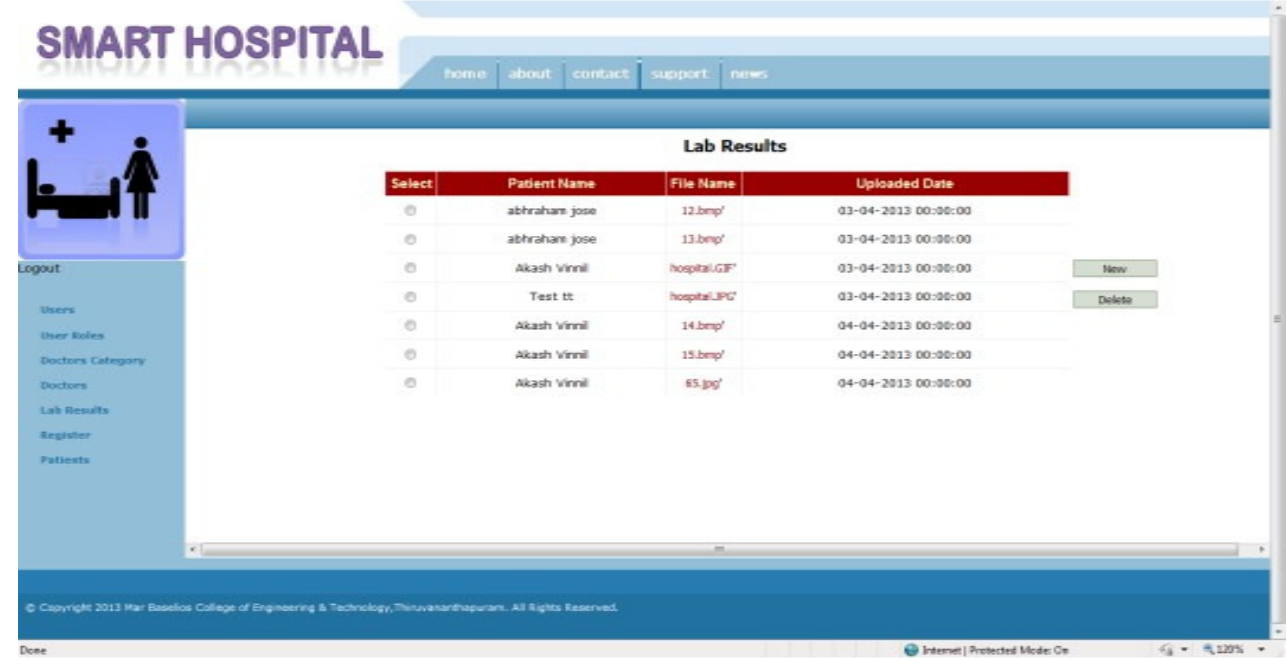

Fig.No.8.ECG results of various patients. 
Health Informatics-An International Journal (HIIJ) Vol.3, No.3,August 2014

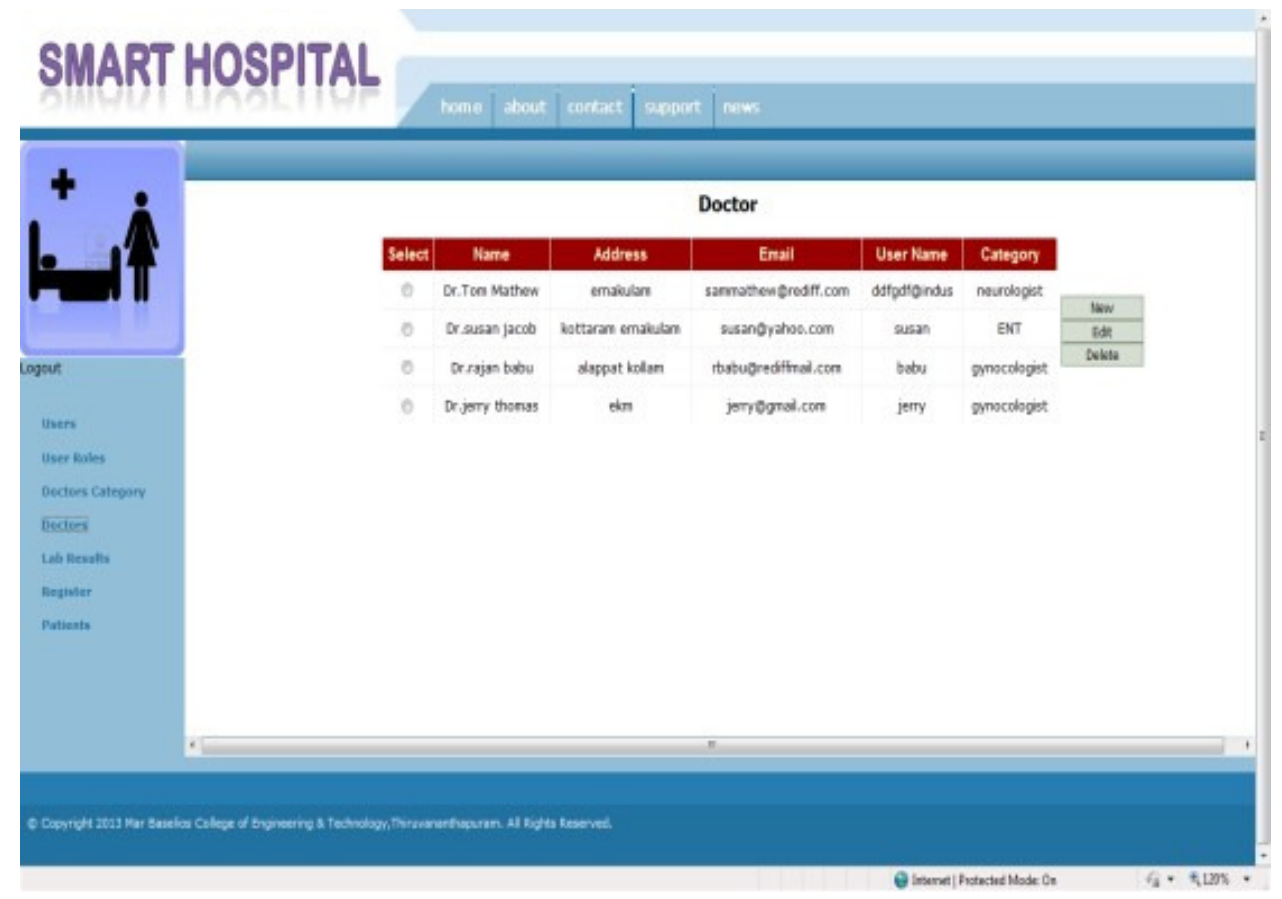

Fig No.9.List of doctors

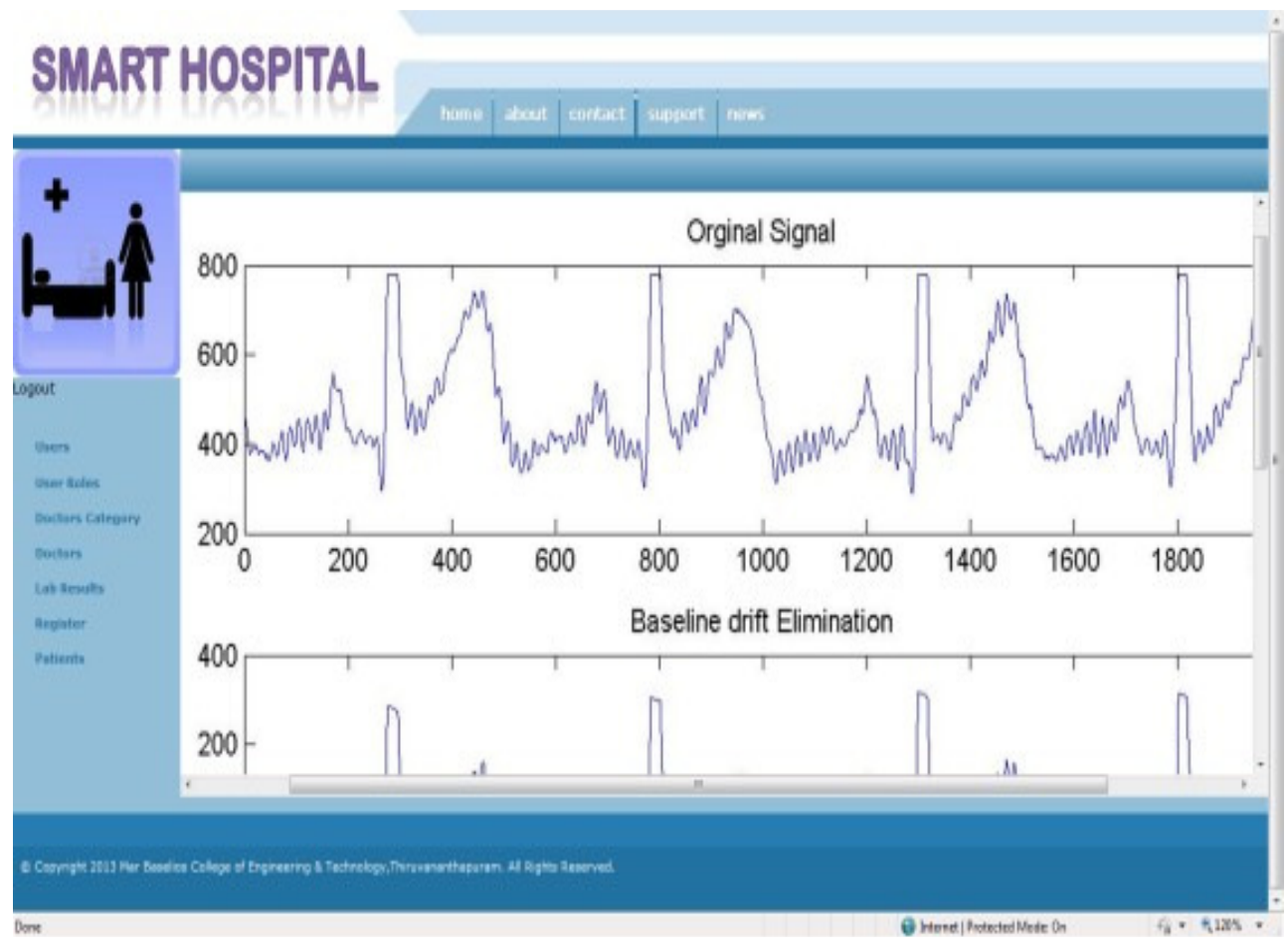

Fig.No.10.ECG image of patient displayed 


\section{Conclusion}

This system is an initiative of automation in critical health care management. It provides continuous monitoring of patient and allows automatic data transfer. The system facilitates equitable access to the specialists for all patients regardless of their location. It provides opportunities for patients and relatives to contact their specialist. The system can completely automate the medical emergency conditions happening in an ICU.

\section{ACKNOWLEDMENT}

The authors would like to thank their colleagues at Mar Baselios College of Engineering and Technology for valuable discussions and feedback, also the reviewers for their constructive comments.

\section{REFERENCES}

[1]Lai Khin Wee, Yeo KeeJiar, EkoSupriyanto "Electrocardiogram Data Capturing System and Computerized Digitization using Image Processing Techniques" International Journal of Biology and Biomedical Engineering Issue 3, Volume 3, 2009.

[2]Ljup`coHad`zievski, Bo`skoBojovic', VladanVuk`cevic', PetarBeli`cev, Sini`saPavlovic', ZoranaVasiljevic'-Pokraj'cic', and MiodragOstojic''A Novel Mobile Transtelephonic System With Synthesized 12-Lead ECG' IEEE Transaction on Information Technology in Biomedicine, Volume 8, 2004.

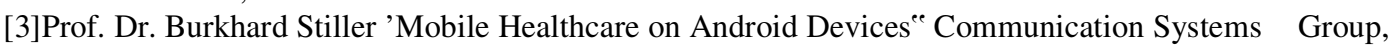
Diploma Thesis, Dept. of Informatics, November 2010.

[4]Karen Panetta, Yicong Zhou,SosAgaian,HongweiJia”Non linearunsharp masking for mammogram enhancement" , IEEE Transaction on Information Technology in Biomedicine Volume. 15, November 2011.

[5]Al-Naima, F. M., A. H. Ali, and S. S. Mahdi. "Data acquisition for myocardial infarction classification based on wavelets and Neural Networks." Systems, Signals and Devices, 2008. IEEE SSD 2008.5th International Multi-Conference on.IEEE, 2008.

[6]Chagas, A. V., E. A. B. Da Silva, and J. Nadal. "ECG data compression using wavelets." Computers in Cardiology 2000.IEEE, 2000. 